\title{
Changing trends in anti-psychotic prescription pattern in Pakistan
}

\author{
Mazhar Malik', M. Usman Ghani², \\ Wardha Mazhar ${ }^{3}$, Nargis Munir ${ }^{4}$
}

\begin{abstract}
Background and Objective: This study was designed to identify the changing trends in Antipsychotic prescription pattern in Pakistan. It was part of the research project Research on East Asian Psychotropic Prescription Pattern (REAP) carried out to identify the prescription patterns of schizophrenic patients in different countries located in Asia. Our objective was to assess the trend and change of psychotropic drug prescriptions for patients with schizophrenia.

Methods: The design of the study was quantitative and of descriptive epidemiology. This study was carried out from $30^{\text {th }}$ March 2017. Data was collected on a unified protocol by the Psychiatrists from Pakistan. Three (3) centers i.e., Lahore, Karachi and Islamabad provided the data. Indoor and outdoor cases with Schizophrenia were recruited. A web based recording system for collection of data done at Taipei Taiwan, and statistical analysis was performed and transferred to all participating centers including Pakistan.

Results: The main findings of the study were that majority of the patients were prescribed antipsychotic poly pharmacy drug. It was also found that Anxiolytics, anti-depressants and Antiparkinsonian drugs were also co-prescribed.

Conclusion: It was concluded that antipsychotic poly pharmacy along with Anxiolytics, anti-depressants and Anti-parkinsonian drugs were prescribed to patients with schizophrenia in Pakistan.
\end{abstract}

KEYWORDS: Polypharmacy, Prescription pattern, Psychotropic drugs, Schizophrenia. Antipsychotics.

doi: https://doi.org/10.12669/pjms.35.3.136

How to cite this:

Malik M, Ghani MU, Mazhar W, Munir N. Changing trends in anti-psychotic prescription pattern in Pakistan. Pak J Med Sci. 2019;35(3):653-657. doi: https://doi.org/10.12669/pjms.35.3.136

This is an Open Access article distributed under the terms of the Creative Commons Attribution License (http://creativecommons.org/licenses/by/3.0), which permits unrestricted use, distribution, and reproduction in any medium, provided the original work is properly cited.

1. Prof. Dr. Mazhar Malik,

Professor of Psychiatry, HoD Department,

2. Dr. M. Usman Ghani,

Assistant Professor of Psychiatry,

3. Dr. Wardha Mazhar,

Assistant Professor of Pharmacology,

FUMC, Islamabad, Pakistan.

4. Nargis Munir,

Clinical Psychologist,

1,2,4: Department of Psychiatry and Behavioural Sciences, Rawal Institute of Health Sciences,

Rawal General and Dental Hospital,

Lehtrar Road, Islamabad, Pakistan.

Correspondence:

Prof. Dr. Mazhar Malik, Professor of Psychiatry,

HoD Department of Psychiatry and Behavioural Sciences, Rawal Institute of Health Sciences, Rawal General and Dental Hospital, Lehtrar Road, Islamabad, Pakistan. Email: mazharm16@hotmail.com

* Received for Publication:

September 15, 2018

* $1^{\text {st }}$ Revision Received:

* $\quad 2^{\text {nd }}$ Revision Received:

* Final Revision Accepted:
December 4, 2018

April 9, 2019

April 12, 2019

\section{INTRODUCTION}

International Collaborative Studies on Psychotropic Prescription, a Research on East Asian Psychotropic Prescription Pattern (REAP) is the largest and the longest international collaborative research project in Asia. REAP undertook large scale surveys on prescription patterns of schizophrenic patients in different countries located in Asia. This research was initially supported by Japan Society for Promotion of Sciences (JSPS).

REAP has conducted four survey studies in Asia. The first survey was carried out in July 2001 in China, Hong Kong, Indonesia, Japan, Korea, Singapore, Malaysia and Taiwan. The objective of this study was to find out the class of antipsychotic drugs used for schizophrenic in patients. The survey in 2001 reported the dominant use of the First-Generation Antipsychotics (FGAs) such 
as Chlorpromazine and Haloperidol over the Second-Generation Antipsychotics (SGAs) such as Quetiapine and Risperidone. To review the change since 2001, the second survey was carried out in July 2004 in mainland China, Hong Kong, Korea, Japan, Taiwan and Singapore that followed the same research protocol and questionnaire. The findings of follow-up survey suggested that the use of SGA's increased sharply in these countries since $2001{ }^{1}$

The fact that anti-depressants prescribed widely to non depressants patients were taken into consideration and the first REAP survey on antidepressant was carried out in March 2004, and studied medical records of patients receiving antidepressants. The results of this survey suggested that one third of patient receiving antidepressants had other diagnosis such as somatoform disorders as well as schizophrenia. ${ }^{2}$

The third survey was carried out in 2008 in China, Korea, Japan, Taiwan, Hong Kong, Singapore, Thailand, Malaysia and India. This survey was also conducted on the use of antipsychotic drugs. With the participation of India, Malaysia and Thailand, REAP became the research group covering almost all the countries of Asia. This survey showed that SGA's are the main antipsychotic drugs for the treatment of Schizophrenia in Asia. ${ }^{3}$ Another survey on the use of antidepressants was conducted in 2014 by East Asia and South Asia. ${ }^{4,5}$

Possible impacts of REAP were for the better understanding and improvement of prescription of antipsychotics in participating countries and contribution to scientific exchange among Asian psychiatrists. It has influenced the prescription pattern in participating countries.

The standard treatments for Schizophrenia during the last 60 years have been First Generation Antipsychotic drugs. The First Generation Antipsychotic drugs (classical or typical) showed a great efficacy at reducing positive symptoms like auditory hallucinations and delusions in Schizophrenia.

Various researches were carried out on Asian Psychotropic Prescription Pattern (REAP) to access antipsychotic medications and psychotropic cotreatments in Asian patients with schizophrenia. ${ }^{6}$ One of the studies was on the use of Clozapine in patients with Schizophrenia. The result of this study showed that the use of Clozapine prescription has considerably decreased over time from 2001 to 2009 in China, while its use has increased in
Korea and Singapore during this period. ${ }^{7}$ Another REAP study was conducted on the prescribing pattern of antipsychotic medications in patients with Schizophrenia in a tertiary care hospital in India. The results showed that $6 \%$ patients were on monotherapy; whereas Asenapine was the only drug used while rests of $94 \%$ patients were on combination therapy. ${ }^{8}$ Another study was conducted on the cardiovascular risks of atypical antipsychotic drug treatment in 2007. According to this study, typical antipsychotics are the drug of choice for patients with Schizophrenia. Atypical antipsychotics are much better than the conventional antipsychotic and do not cause extra pyramidal symptoms. Another study was conducted on the use of antipsychotic polypharmacy in patients with Schizophrenia. The major findings of this study were that multiple antipsychotics are widely prescribed to more than one third of the schizophrenic patients in Asian region. In addition, Antipsychotic PolyPharmacy (APP) prescription pattern decreased in Asia from $46.9 \%$ in 2001 to $38.3 \%$ in 2004 , but then increased to $42.9 \%$ in 2009. The frequency of Antipsychotic Poly Pharmacy in the current study is in the range of previous findings. ${ }^{9}$ The objective of current study was to assess the trend and change of psychotropic drug prescriptions for patients with schizophrenia.

\section{METHODS}

Research Design: The design of the study was quantitative and of descriptive epidemiology. Data was collected on a unified protocol by the Psychiatrists of the participating countries from Pakistan. The data collection for the current study was started from $30^{\text {th }}$ March 2017. Initially Five (5) centers were selected for data collection, while three (3) centers i.e., Lahore, Karachi and Islamabad provided the data. Indoor and outdoor cases with Schizophrenia were recruited. The prescribing patterns of psychiatrists were analyzed, taking consideration of the available psychotropic drugs on the market and different geographical and practicing systems. The data was collected on the short-form version of the questionnaire. Demographic details like age, gender, duration of illness, rural urban setting, were taken before data collection.

\section{Inclusion criteria:}

1. Diagnosed patients with Schizophrenia according to Diagnostic and Statistical Manual 5 (DSM 5) or International Classification of Diseases (ICD- 10). 
Table-I: Demographic details of patients.

\begin{tabular}{lcccc}
\hline Variables & Total N=298 & $\begin{array}{c}\text { Centre Case 246 } \\
\text { (Lahore) }\end{array}$ & $\begin{array}{c}\text { Centre Case 13 } \\
\text { (Karachi) }\end{array}$ & $\begin{array}{c}\text { Centre Case 39 } \\
\text { (Islamabad) }\end{array}$ \\
\hline $\begin{array}{l}\text { Age (20-65 years) } \\
\text { Mean= SD }\end{array}$ & 36.9 & 36.6 & & \\
Gender $\boldsymbol{n}$ (\%) & & & 41.2 & 37.0 \\
Male & $168(56.4 \%)$ & $138(56 \%)$ & $8(61.5 \%)$ & $22(56.4 \%)$ \\
Female & $130(43.6 \%)$ & $108(43.9 \%)$ & $5(38.5 \%)$ & $17(43.6 \%)$ \\
Treatment setting $\boldsymbol{n}(\boldsymbol{0})$ & & & & \\
Outpatient & $156(52.3 \%)$ & $108(43.9 \%)$ & $11(84.6 \%)$ & $37(94.9 \%)$ \\
Inpatient & $142(47.7 \%)$ & $138(56.1 \%)$ & $2(15.4 \%)$ & $2(5.1 \%)$ \\
Acute cases & $49(31.4 \%)$ & $46(42.6 \%)$ & & $3(8.1 \%)$ \\
Chronic cases & $12(37.5 \%)$ & $11(55.0 \%)$ & & $1(100.0 \%)$ \\
\hline
\end{tabular}

2. Being a patient on the day of survey.

3. Willingness to participate in the study. Exclusion criteria:

1. The patients who were not willing to participate were excluded.

2. Presence of serious illness, for example serious cardiovascular illness, recent history of stroke, hepatic failure and renal failure.

Data Collection and Analysis: Ethical permission was taken from the administration of the Rawal Institute of Health Sciences Islamabad Pakistan, before collecting the data. Data was collected by the psychiatrist in front of patient.

Informed consent was taken from the patients for participating in the REAP survey before the collection of data. The data was collected through the questionnaire which includes demographic details, psychiatric symptoms, psychotropic medications, co morbidities, physical conditions, and adverse drug reactions, considering different geographical and practicing systems and available psychotropic drugs in the market. Diagnosed cases of schizophrenia were the population of the study and all those patients meeting the criteria were the sample of study. Nonrandom, purposive sampling technique was used. The questionnaire used for data collection was a reliable and validated tool for the patients of schizophrenia. Duration of acute and chronic illness was taken while collecting data.

This is a web based recording system for collection of data done at Taipei Taiwan, and statistical analysis was performed and transferred to all participating centers including Pakistan.

\section{RESULTS}

The demographic details from three centers of Pakistan are given in Table-I. The demographic characteristics included age, gender, treatment setting, and acute and chronic cases. Table-II shows percentage of patients on typical, atypical and on long acting antipsychotics. Table-III shows co prescription of other drugs along with the antipsychotics while Table-IV shows top ten antipsychotics and co prescription of other drugs.

\section{DISCUSSION}

Schizophrenia is a psychotic illness with a range of clinical symptoms including auditory hallucinations, delusions and anhedonia that

Table-II: Typical and Atypical Antipsychotics.

\begin{tabular}{llcc}
\hline Class of Drugs & Drug Name & $N=298$ & $\%$ \\
\hline Typical Anti-psychotic drugs & & \\
& Haloperidol & 79 & $26.5 \%$ \\
& Trifluoperazine & 17 & $5.7 \%$ \\
& Clopenthixol & 1 & $0.3 \%$ \\
& Chlorpromazine & 1 & $0.3 \%$ \\
Atypical Anti-psychotic drugs & & \\
$\quad$ Risperidone & 186 & $62.4 \%$ \\
& Quetiapine & 55 & $18.5 \%$ \\
& Clozapine & 33 & $11.1 \%$ \\
& Aripiprazole & 18 & $6.0 \%$ \\
& Olanzapine & 18 & $6.0 \%$ \\
Poly pharmacy & Amisulpride & 2 & $0.7 \%$ \\
(prescription of & Typical and a typical & 151 & $51.7 \%$ \\
more than one & antipsychotics & & \\
antipsychotic drug) & & & \\
Long acting- & Fluphenazine & & \\
antipsychotic & decanoate & 38 & $12.8 \%$ \\
& Zuclopenthixol & 20 & $6.7 \%$ \\
& decanoate & & \\
& Flupentixol decanoate & 8 & $2.7 \%$ \\
& Haloperidol decanoate & 8 & $2.7 \%$ \\
\hline
\end{tabular}


Table-III: Co-prescription of other medications.

\begin{tabular}{llcc}
\hline Mood-Stabilizer & & & \\
& Valproic acid & 58 & $19.5 \%$ \\
& Carbamazepine & 15 & $5.0 \%$ \\
& Lamotrigine & 6 & $2.0 \%$ \\
& Lithium & 2 & $0.7 \%$ \\
& Oxcarbazepine & 2 & $0.7 \%$ \\
Anxiolytic & Clonazepam & 116 & $38.9 \%$ \\
& Lorazepam & 36 & $12.1 \%$ \\
& Oxazepam & 10 & $3.4 \%$ \\
& Alprazolam & 8 & $2.7 \%$ \\
Bypnotics used & Bromazepam & 6 & $2.0 \%$ \\
Anti-depressants used & 21 & $7 \%$ \\
Anti parkinsonian drug used & 56 & $18.8 \%$ \\
\hline
\end{tabular}

coalesce to form a devitalizing syndrome affecting all functional domains. These symptoms respond in different manner to treatment depending on various factors. A study conducted on the pattern of use of antipsychotic drugs found that $31 \%$ of the patients received a combination of atypical and typical antipsychotics in greater than maximum recommended doses. ${ }^{10}$

The treatment of Schizophrenia has been challenging despite advancing pharmacological treatment which often leads to the use of two or more antipsychotics to treat refractory and residual psychotic signs and symptoms. A systematic review with a pooling data across four decades from 1979 to 2009 found the median rate for the prescription of antipsychotic polypharmacy to be $19.6 \%$ globally and $32 \%$ in Asia which was higher as compared to other regions such as North America and Oceania. ${ }^{11}$

Another study on relation of antipsychotic polypharmacy and quality of life in patients with Schizophrenia in China suggested that the rate of APP prescription was 31 with $89 \%$ on two antipsychotics and $10.4 \%$ received three or more antipsychotics. It also showed patients prescribed on APP were mainly on higher doses of SGAs along with anticholinergics. ${ }^{12}$

The current study has been conducted to assess the trends and changes in psychotropic drug prescriptions for patients with Schizophrenia. The findings suggested that the use of typical and typical antipsychotic drugs is $15.3 \%$ and $31.6 \%$ respectively, whereas the use of polypharmacy is $51.7 \%$. Co-prescription pattern of other drugs showed that $77 \%$ mood stabilizers, $56 \%$ antidepressants, $57.5 \%$ anxiolytics, $7 \%$ hypnotics and $65 \%$ antiparkinsonian drugs are being given to the patients on top with antipsychotics. These
Table-IV: List of top ten antipsychotics and commonly prescribed mood stabilizers, anxiolytics and anti parkinsonian drugs.

\begin{tabular}{clcc}
\hline $\begin{array}{c}\text { Antipsyhotics } \\
\text { (Top ten used) }\end{array}$ & Drug Name & $N$ & $\%$ \\
\hline & Risperidone & 186 & $62.4 \%$ \\
& Haloperidol & 79 & $26.5 \%$ \\
& Quetiapine & 55 & $18.5 \%$ \\
& Fluphenazine decanoate & 38 & $12.8 \%$ \\
& Clozapine & 33 & $11.1 \%$ \\
& Zulcopenthixol decanoate & 20 & $6.7 \%$ \\
& Aripiprazole & 18 & $6.0 \%$ \\
& Olanzapine & 18 & $6.0 \%$ \\
& Trifluoperazine & 17 & $5.7 \%$ \\
Antiparkinsonian drugs & 207 & $65.9 \%$ \\
Other drugs & & \\
(Top ten used) & Anxiolytic(Clonazepam) & 116 & $38.9 \%$ \\
& Mood Stabiliser & 58 & $19.5 \%$ \\
$\quad$ (Valproic Acid) & & \\
& Antidepressants & 56 & $18.8 \%$ \\
Anxiolytic(Lorazepm) & 36 & $12.1 \%$ \\
Hypnotics & 21 & $7 \%$ \\
Mood Stabilisers & 15 & $5.0 \%$ \\
$\quad$ (Carbamezipine) & & \\
\hline & &
\end{tabular}

findings regarding polypharmacy are in line with a similar study conducted in 2014 in a developing country which showed $31 \%$ of patients were receiving a combination of typical and atypical antipsychotics. ${ }^{13}$

Another study conducted in Pakistan suggested that the pharmacotherapy of patients consisted mainly of antipsychotic drugs i.e.; $86 \%$. Other drugs that were prescribed included anxiolytics, antiparkinsonian drugs, anticonvulsants and antidepressants. The results regarding antipsychotic drug use are consistent with our study showing $50 \%$ of patients taking typical antipsychotics and $56 \%$ on atypical antipsychotics. ${ }^{14}$

Study Limitations: Data has been taken from three centres of Pakistani, Karachi, Lahore, and Islamabad only. Data details from other centers were not provided.

\section{CONCLUSION}

It was concluded that typical and atypical antipsychotic poly pharmacy were prescribed to patients with schizophrenia in Pakistan. It was also concluded that mood stabilizers, antidepressants, anxiolytics, hypnotics and antiparkinsonian drugs are being given to the patients on top with antipsychotics. 
Acknowledgement: We would like to thank the pioneers of this research project, the coordinators Prof. Dr. Naotaka and Dr. Tan Chay Hoon who have been a constant source of inspiration, support and invaluable inputs during our endeavor to complete this project. Thanks to Lahore and Karachi centre who participated in the study. Dr. Shu-YuYang and Dr. Lian $\mathrm{Yu}$ Chen who assisted us in data management.

\section{REFERENCES}

1. Sim K, Su HC, Fujii S, Yang SY, Chong MY, Ungvari $\mathrm{G}$, et al. High-dose antipsychotic use in schizophrenia: a comparison between the 2001 and 2004 Research on East Asia Psychotropic Prescription (REAP) studies. Br J Clin Pharmacol. 2009;67(1):110-117. doi: 10.1111/j.1365-2125.2008.03304.x.

2. Fujii S, Kaneko N, Kitsukawa H. Prescription of Antidepressants in Japan-Finding from International Collaboratinve Study on the Prescription of Antidepressantgs in Five Countries and Areas in Asia. Japanese J Soc Psychiatry. 2005;14:30-35. (In Japanese).

3. Sim K, Yong KH, Chan YH, Tor PC, Xiang YT, Wang CY, et al. Adjunctive mood stabilizer treatment for hospitalized schizophrenia patients: Asia psychotropicprescription study (20012008). Int J Neuropsychopharmacol. 2011;14(9):11571164. doi: $10.1017 /$ S1461145711000563.

4. Chee KY, Tripathi A, Avasthi A, Chong MY, Xiang YT, Sim $K$ et al. Prescribing Pattern of Antidepressants in Children and Adolescents: Findings from the Research on Asia. Psychotropic Prescription Pattern. East Asian Arch Psychiatry. 2016;26(1):10-17.

5. Tripathi A, Avasthi A, Desousa A, Bhagabati D, Shah N, Kallivayalil RA, et al. Prescription pattern of antidepressants in five tertiary care psychiatric centres of India. Indian J Med Res. 2016;143(4):507-513. doi: 10.4103/0971-5916.184289.

6. Xiang YT, Ungvari GS, Correll CU, Chiu HF, Shinfuku $\mathrm{N}$. Trends in the access to and the use of antipsychotic medications and psychotropic co-treatments in Asian patients with schizophrenia. Epidemiol Psychiatr Sci. 2016;25(1):9-17. doi: 10.1017/S2045796015000694.

7. Xiang YT, Wang CY, Si TM, Lee EH, He YL, Ungvari GS, et al. Clozapine use in schizophrenia: findings of the Research on Asia Psychotropic Prescription (REAP) studies from 2001 to 2009. Aust N Z J Psychiatry. 2011;45(11):968-975. doi: 10.3109/00048674.2011.607426.
8. Ito $\mathrm{H}$, Okumura $\mathrm{Y}$, Higuchi $\mathrm{T}$, Tan $\mathrm{CH}$, Shinfuku $\mathrm{N}$. International Variation in antipsychotic prescribing for schizophrenia: Pooled results from the research on East Asia psychotropic prescription (REAP) studies. O J Psych. 2012;2:340-346. doi: 10.4236/ojpsych.2012.224048.

9. Nakano W, Yang Shu-Yu , Fujii S. Characteristics of pharmacotherapy for schizophrenic inpatients in Japan Findings from Research on East Asia Psychotropic Prescription Pattern of Antipsychotics. Rinsyou Seishin Yakuri. 2010;13:103-113.

10. Kongsakon R, Trinidad-Onate P, Chaudhry HR, Raza SB, Leynes CR, Khan IU, et al. Asian outpatients with schizophrenia: a double-blind randomized comparison of quality of life and clinical outcomes for patients treated with olanzapine or haloperidol. J Med Assoc Thai. 2006;89(8):1157-1170.

11. Kim HY, Lee HW, Jung SH, Kang MH, Bae JN, Lee JS, et al. Prescription patterns for patients with schizophrenia in Korea: a focus on antipsychotic polypharmacy. Clin Psychopharmacol Neurosci. 2014;12(2):128-136. doi: 10.9758/cpn.2014.12.2.128.

12. Hou CL, Ma XR, Zang Y, Jia FJ, Lin YQ, Chiu HF, et al. Antipsychotic polypharmacy and quality of life in patients with schizophrenia treated in primary care in China. Int J Clin Pharmacol Ther. 2016;54(1):36-42. doi: 10.5414/ CP202413.

13. Ramadas S, Kuttichira P. A Study of an Antipsychotic Prescription Patterns for Patients with Schizophrenia in a Developing Country. Indian Psychol Med. 2010;32(1):13-16.

14. Fatima A, Barkat U, Mahmood KT. Schizophrenia in Elderly Patients. J Pharm Sci Res. 2011;3(1):952-960.

\section{Authors' Contribution}

MM: Conception and design.

MUG \& WM: Collection and assembly of data.

NM \& MUG: Analysis and interpretation of the data.

MM, NM \& WM: Drafting of the article.

MM \& MUG: Critical revision of the article for important intellectual content.

NM \& MUG: Statistical expertise.

MM: Final approval and guarantor of the article. 